\title{
Analyse de la flore fongique de la carotte conservée au froid : prépondérance de Mycocentrospora acerina (Hartig) Deighton
}

\author{
B Le Cam ${ }^{1}$, F Rouxel $^{1^{*}}$, F Villeneuve 2 \\ ${ }^{1}$ INRA, station de Pathologie végétale, BP 29, F-35650 Le Rheu; \\ ${ }^{2}$ Ctifl-Sileban, 50760 Barfleur, France
}

(Reçu le 28 mai 1992; accepté le 13 octobre 1992)

\begin{abstract}
Résumé - La conservation des carottes au froid après récolte se développe depuis peu en France; l'objectif de cette étude est d'inventorier les agents pathogènes susceptibles de provoquer des dégâts dans cet environnement particulier et de définir les conditions optimales de stockage. L'analyse de la mycoflore réalisée sur racines de carottes stockées 50 ou 100 j au froid montre que dans les conditions de production et de conservation de la région de Normandie, les principaux problèmes parasitaires rencontrés sont dus à Mycocentrospora acerina et à Phytophthora megasperma; les dégâts occasionnés par Botrytis cinerea, Sclerotinia sclerotiorum et Stemphylium radicinum sont d'importance mineure. Par ailleurs, l'incidence des conditions de conservation est démontrée : les carottes stockées au froid humide $\left(0,5\right.$ à $1{ }^{\circ} \mathrm{C}-98 \%$ d'humidité relative) présentent un pourcentage d'attaque plus faible que celles maintenues au froid conventionnel $\left(2{ }^{\circ} \mathrm{C}-85-90 \%\right.$ d'humidité relative). La caractérisation biologique des différents agents pathogènes isolés indique que seuls $M$ acerina et $B$ cinerea sont capables de provoquer des symptômes dès $0,5^{\circ} \mathrm{C}$. Le comportement de l'ensemble des parasites en relation avec la température et l'hygrométrie est discuté. L'intérêt d'un délai entre l'arrachage et la mise en conservation sur le développement des maladies et plus particulièrement sur $M$ acerina est également mis en évidence; l'intensité d'attaque de ce parasite, dans les conditions optimales de conservation pour la carotte, reste toutefois considérable et justifie une étude sur la recherche d'une technique de lutte adaptée s'appuyant notamment sur la spécificité des relations hôte-parasite.
\end{abstract}

Daucus carota $=$ carotte $/$ pathologie post-récolte $/$ Mycocentrospora acerina / conservation au froid

Summary - Post-harvest pathogens on cold stored carrots: Mycocentrospora acerina (Deighton), the major spoilage fungus. A survey was conducted to inventory pathogens likely to attack carrot roots stored under cold conditions. An analysis of the carrot root mycoflora under storage and production conditions in Normandy, France, showed that major fungal spoilage was due to Mycocentrospora acerina and Phytophthora megasperma; rot caused by Botrytis cinerea, Sclerotinia sclerotiorum and Stemphylium radicinum was only of secondary importance. The study also revealed that storage losses due to fungal spoilage were lower in ice-bank cooled stores at $0.5-1{ }^{\circ} \mathrm{C}$, 98\% relative humidity $(\mathrm{RH})$ than in conventionally cooled stores at $2{ }^{\circ} \mathrm{C}, 85-90 \% \mathrm{RH}$. Only $\mathrm{M}$ acerina and $\mathrm{B}$ cinerea strains were pathogenic on carrot root slices at temperatures as low as $0.5{ }^{\circ} \mathrm{C}$, but were not particularly adaptated for cold conditions. The behaviour of the pathogens related to temperature and relative humidity is discussed. This study showed the existence of a relationship between the healing of wounds in carrot roots and infection by $\mathrm{M}$ acerina by exposing them before cold storage. Nevertheless, spoilage wastage caused by this pathogen still remains important even under optimal storage conditions for carrot crops.

Daucus carota / post-harvest diseases / Mycocentrospora acerina / cold storage

* Correspondance et tirés à part 


\section{INTRODUCTION}

En France, la carotte destinée à la consommation hivernale était jusqu'à présent conservée dans le sol. Cette technique a permis à la Normandie de devenir la première région française productrice de carottes mais les conditions climatiques hivernales posent de nombreux problèmes : difficultés d'accès au champ, détérioration de la structure du sol à la récolte, lourdes pertes dues aux nécroses et pourritures hivernales (Breton et Rouxel, 1985a; Montfort et Rouxel, 1986), reprise de la végétation au printemps; ainsi entre avril et juin, ce type de production ne permet plus de proposer aux consommateurs un produit de qualité en quantité suffisante.

Afin de pallier ces difficultés et de répondre aux exigences du marché actuel, les professionnels s'orientent depuis peu vers la conservation au froid après récolte (température de $0,5-1^{\circ} \mathrm{C}$, $98 \%$ d'humidité relative). Cette nouvelle forme de gestion de la conservation permettra sans doute de s'affranchir des principaux problèmes liés au sol; toutefois la littérature étrangère signale plusieurs agents pathogènes capables de se développer en chambres froides et susceptibles de provoquer certaines années de très lourdes pertes : Rhizoctonia carotae, signalé il $y$ a plus de 40 ans aux USA sur carottes stockées (Rader, 1948), et plus récemment en Suède (Ramert, 1984) et au Canada (Punja, 1987), a occasionné des pertes de l'ordre de 50-70\% au Danemark (Jensen, 1969). Mycocentrospora acerina, un autre champignon phytopathogène, est considéré en Angleterre et en Norvège comme une des limites à la conservation au froid (Derbyshire et Crisp, 1971; 1978; Hoftun, 1985). Enfin, d'autres parasites plus polyphages se manifestent également dans ces mêmes conditions: c'est le cas de Botrytis cinerea (Tahvonen, 1985; Geeson et al, 1988; Tronsmo, 1989), et de Sclerotinia sclerotiorum (Lewis et Garrod, 1983; Geeson et al, 1988).

Dans la perspective d'un développement du stockage de la carotte au froid en France, cet article dresse un inventaire des problèmes parasitaires susceptibles de se manifester sur carottes après récolte en fonction de nos conditions de culture et de conservation; il précise, par ailleurs, quelques conditions de manifestation des principaux agents pathogènes en cause.

\section{MATÉRIEL ET MÉTHODES}

\section{Étude de l'évolution de l'état sanitaire des carottes en cours de conservation}

\section{Influence des conditions de conservation au froid}

Deux types de froid ont été comparés dans 2 salles de conservation ( $63 \mathrm{~m}^{3}$ chacune) fonctionnant à la station expérimentale du Sileban à Barfleur (50) :

- le froid conventionnel $\left(2{ }^{\circ} \mathrm{C}, 85-90 \%\right.$ d'humidité relative) où l'hygrométrie est maintenue à l'aide d'un humidificateur humidisonique (débit de l'air : $7800 \mathrm{~m}^{3} / \mathrm{h}$ ),

- le froid humide $\left(0,5-1{ }^{\circ} \mathrm{C}, 98 \%\right.$ d'humidité relative) produit par une tour de refroidissement de marque Baltimore (débit de l'air : $6300 \mathrm{~m}^{3} / \mathrm{h}$ ).

Des racines de carotte (variété Nandor), provenant d'une culture âgée de 7 mois, récoltées le 16 janvier 1990 sur une parcelle homogène de la zone légumière du Val de Saire, sont triées pour ne conserver que celles d'aspect sain, réparties en sacs de polyéthylène non perforés contenant chacun 100 racines, puis immédiatement entreposées au froid à raison de 6 sacs dans chaque salle de conservation. Ces dernières sont alors complétées par des lots hors essai afin de permettre un flux normal de l'air.

\section{Influence du délai entre l'arrachage des racines et la mise en conservation}

Un deuxième lot de carottes récoltées et conditionnées comme précédemment provenant d'une parcelle «à risque" (culture répétée de carottes) est placé en salle de conservation au froid humide $0,6,12,24$ et $36 \mathrm{~h}$ après arrachage (le 10 janvier 1990). Pendant la phase transitoire, les racines déposées dans des containers ajourés $\left(1 \mathrm{~m}^{3}\right)$ sont entreposées dans un local aéré à $5-7^{\circ} \mathrm{C}$.

\section{Évaluation de l'état sanitaire}

Pour les 2 expériences, l'état sanitaire des carottes a été analysé après 2 périodes de conservation : 50 et $100 \mathrm{j}$. Chaque notation est réalisée sur 3 répétitions (3 sacs de 100 carottes) par répartition des racines préalablement lavées en saines et malades, tous symptômes parasitaires confondus. Les résultats, exprimant les pourcentages de carottes malades en fonction de chaque modalité, sont présentés pour les deux dates de sortie.

\section{Analyse des symptômes et de la flore fongique associée aux racines malades}

Ces analyses ont porté sur les racines malades de l'essai étudiant l'influence du délai entre l'arrachage 
des racines et la mise en conservation (Protocole cidessus). Pour chaque modalité, les carottes sont répertoriées en fonction des types de symptômes; une appréciation visuelle confirmée par un examen microscopique a suffi pour identifier les dégâts dus à plusieurs parasites classiques: Sclerotinia sclerotiorum, Botrytis cinerea, Rhizoctonia violacea.

Dans les autres cas de pourritures et nécroses moins caractéristiques, des isolements microbiologiques ont été pratiqués selon le protocole suivant : sur chaque racine à analyser, des tissus épidermiques sont prélevés en bordure de la zone nécrosée; chaque échantillon est désinfecté superficiellement dans une solution d'éthanol à $95 \%$ pendant $30 \mathrm{~s}$, rincé à l'eau stérile puis séché sur papier filtre. Dix fragments de quelques $\mathrm{mm}$ de longueur sont alors déposés en boîtes de Petri contenant un milieu nutritif gélosé à base de malt additionné soit de streptomycine $(0,25 \%$ o), soit du complexe polymixine - pénicilline (respectivement $0,025 \%$ et $0,25 \%$ ). Cette opération est répétée 2 fois pour chaque milieu. Après 5 à $7 \mathrm{j}$ d'incubation à $20^{\circ} \mathrm{C}$, les colonies développées sont identifiées. Les résultats présentés expriment la part relative des principales espèces fongiques associées aux différents symptômes après 50 et 100 j de conservation.

\section{Étude du pouvoir pathogène des principales espèces fongiques inventoriées sur carotte en cours de conservation}

\section{Matériel étudié}

L'étude a porté sur 20 isolats fongiques appartenant à 10 genres (tableau 1). Seize proviennent de carottes malades stockées au froid; ils sont comparés à 4 autres issus de carottes conservées au champ, de tournesol et de féverole.

\section{Test d'agressivité sur carotte}

La technique utilisée, identique pour l'ensemble des isolats, est inspirée de celle mise au point par Montfort et Rouxel (1988). Des racines d'aspect sain, n'ayant pas subi de traitement fongicide, sont soi-

Tableau I. Liste et origine des isolats étudiés pour leur agressivité sur carotte.

Code Genre, espèce Origine et conditions d'isolement Plante hôte $\begin{array}{r}\text { Année } \\ \text { d'isolement }\end{array}$

\begin{tabular}{|c|c|c|c|c|c|}
\hline 1 My & Mycocentrospora acerina & Barfleur & (50) - champ & carotte & 1990 \\
\hline 2 My & " & Barfleur & $(50)$ - froid & $"$ & " \\
\hline 3 My & $"$ & Nantes & (44) - froid & $"$ & $"$ \\
\hline $4 \mathrm{My}$ & $"$ & Barfleur & $(50)-$ froid & $"$ & $"$ \\
\hline 5 Bo & Botrytis cinerea & - & (22) - champ & féverole & 1986 \\
\hline $6 \mathrm{Bo}$ & " " & Barfleur & $(50)-$ froid & carotte & 1990 \\
\hline 7 Bo & $"$ & Nantes & (44) - froid & " & 1990 \\
\hline 8 Bo & $"$ & Barfleur & $(50)-$ froid & $"$ & 1990 \\
\hline $9 \mathrm{Sc}$ & Sclerotinia sclerotiorum & Francheville & (51) - champ & tournesol & 1987 \\
\hline $0 \mathrm{Sc}$ & " $\quad$ " & Barfleur & $(50)-$ froid & carotte & 1990 \\
\hline $1 \mathrm{Fr}$ & Fusarium roseum var arthrosporioides & Barfleur & $(50)-$ froid & carotte & 1990 \\
\hline $2 \mathrm{Fr}$ & " n $\quad$ " & Barfleur & $(50)$ - froid & " & 1990 \\
\hline $3 \mathrm{St}$ & Stemphylium radicinum & Barfleur & (50) - champ & carotte & 1987 \\
\hline 4 St & $" 1$ & Barfleur & $(50)-$ froid & $"$ & 1990 \\
\hline $5 \mathrm{Pm}$ & Phytophthora megasperma & Barfleur & $(50)$ - froid & carotte & 1990 \\
\hline $6 \mathrm{Al}$ & Alternaria sp & Barfleur & $(50)$ - froid & carotte & 1990 \\
\hline $7 \mathrm{Al}$ & " & Barfleur & $(50)-$ froid & " & 1990 \\
\hline $8 \mathrm{Cy}$ & Cylindrocarpon sp & Barfleur & $(50)$ - froid & carotte & 1990 \\
\hline $9 \mathrm{Ph}$ & Phoma exigua var exigua & Barfleur & $(50)-$ froid & carotte & 1990 \\
\hline 0 Ps & Phomopsis sp & Barfleur & (50) - froid & carotte & 1990 \\
\hline
\end{tabular}


gneusement lavées puis découpées en demi-tronçons d'environ $3 \mathrm{~cm}$ de longueur. Après blessure de l'épiderme à l'aide d'une toile abrasive, les demi-tronçons sont placés dans une chambre humide à raison de 27 par traitement. Sur 25 d'entre eux, un explantat calibré (diamètre $5 \mathrm{~mm}$ ) d'une pré-culture du champignon à étudier est déposé; les 2 autres demi-tronçons constituant les témoins reçoivent une pastille gélosée vierge.

Les chambres humides sont ensuite placées aux températures suivantes : $0,5,5,10$ et $15^{\circ} \mathrm{C}$. Une notation, consistant à mesurer les dimensions des nécroses autour de chaque point d'inoculation, permet alors d'exprimer les résultats en surface moyenne d'attaque $\left(\mathrm{mm}^{2}\right)$ après 6 jours d'incubation pour chaque température et isolat étudiés.

\section{RÉSULTATS}

\section{Mise en évidence de l'incidence des conditions de conservation sur l'état sanitaire des carottes}

\section{Effet du type de froid}

À $50 \mathrm{j}$ de conservation, les carottes stockées au froid conventionnel présentent plus d'attaques parasitaires que celles conservées au froid humide : $15 \%$ de racines malades contre $3,5 \%$ (fig 1). À $100 \mathrm{j}$, cette différence est nettement confirmée avec une forte dégradation de l'état sanitaire global, les pertes s'élevant à $20 \%$ après $100 \mathrm{j}$ de stockage au froid humide.

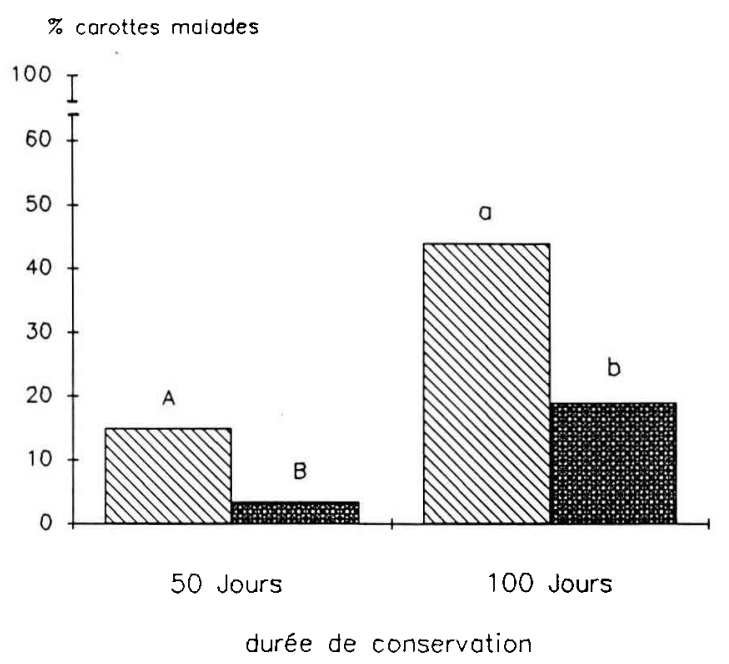

Fig 1. Pourcentage de carottes malades à 50 ou $100 \mathrm{j}$ de conservation au froid conventionnel $V .\left(2{ }^{\circ} \mathrm{C}, 85-90 \%\right.$ d'humidité relative) ou au froid humide $\left(0,5-1{ }^{\circ} \mathrm{C}, 98 \%\right.$ d'humidité relative). Les histogrammes affectés d'une même lettre ne sont pas significativement différents pour $p=0,05$ (Test de Newman-Keuls).

\section{Effet de la durée du délai «arrachage des racines-mise en conservation"}

À $50 \mathrm{j}$ de conservation, l'état sanitaire global demeure bon $(<10 \%$ de carottes malades) quel que soit le délai «arrachage-mise en conservation"; la réduction des attaques reste insuffisante pour être significative (fig 2).

Par contre, à 100 j de stockage, la situation sanitaire se détériore nettement mais l'effet $d u$ délai "arrachage-mise en conservation" est clairement mis en évidence avec des taux d'attaques voisins de $60 \%$ et $50 \%$ pour des délais respectifs de 12 et $36 \mathrm{~h}$ contre $91 \%$ pour le témoin.

\section{Mycocentrospora acerina : principal constituant de la mycoflore associée aux carottes malades}

Une analyse fine des symptômes associée à des isolements microbiologiques nous indique que Mycocentrospora acerina constitue le représentant dominant de la flore fongique présente sur carottes malades (fig 3). En effet, dans nos conditions expérimentales, au moins $70 \%$ des symptômes sont attribués à ce champignon dont les dégâts sur carotte se manifestent par des lésions noires bien délimitées que les anglosaxons qualifient de "liquorice rot" (fig 4A).

Phytophthora megasperma, agent de la maladie de la bague bien connue au champ, est à l'origine de $2-30 \%$ des symptômes observés sur

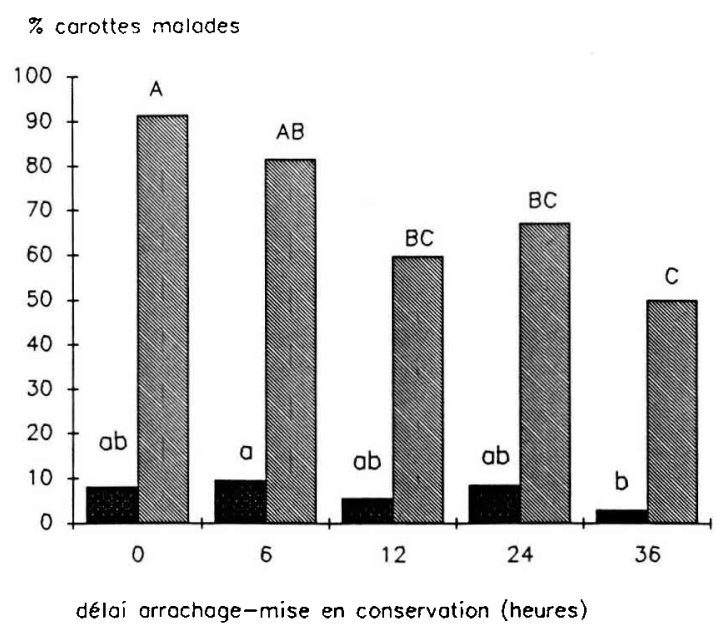

Fig 2. Pourcentage de carottes malades à 50 ou $100 \mathrm{j} \psi$ de conservation au froid humide $\left(0,5-1{ }^{\circ} \mathrm{C}, 98 \%\right.$ d'humidité relative) en fonction du délai arrachage-mise en conservation. Les histogrammes affectés d'une même lettre ne sont pas significativement différents pour $p=0,05$ (Test de Newman-Keuls) 


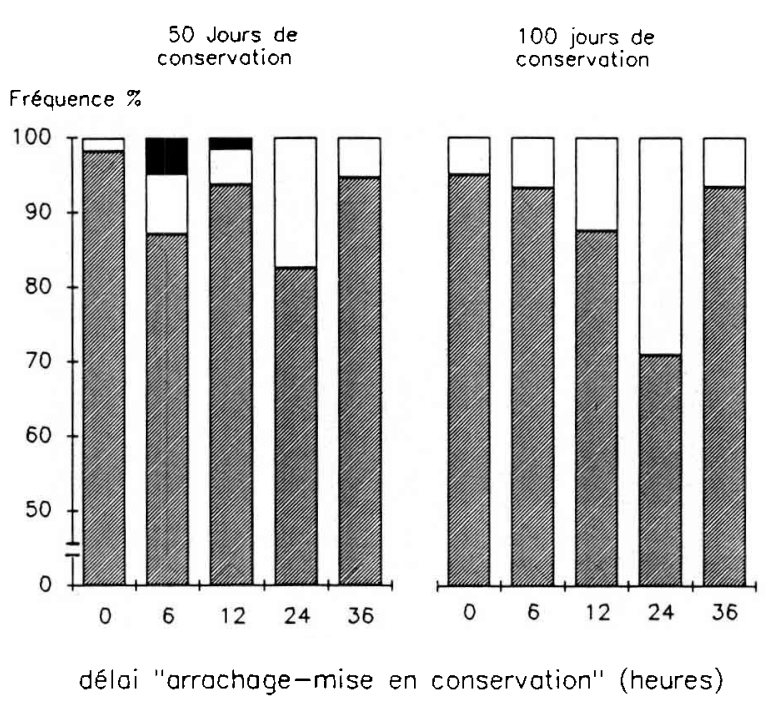

Fig 3. Part relative des principales espèces fongiques associées aux symptômes observés sur carottes à 50 et 100 j de conservation au froid humide en fonction du délai "arrachage-mise en conservation". Mycocentrospora acerina $\mathcal{Z}$, Phytophthora megaspermal , Autres $\square$ : $S$ radicinum, $B$ cinerea, $S$ sclerotiorum, $A$ violacea, $F$ roseum.

carottes stockées après récolte; ces racines présentent des anneaux noirs caractéristiques évoluant en pourriture molle (fig 4B).

Les autres champignons sont présents à une fréquence extrêmement faible, voire absente après $100 \mathrm{j}$ de conservation : il s'agit de Botrytis cinerea, agent de la pourriture grise (fig 4C), Stemphylium radicinum (fig 4D), Sclerotinia sclerotiorum, responsable de la pourriture blanche (fig 4E), Fusarium roseum et Rhizoctonia violacea, et enfin Cylindrocarpon sp, Phomopsis sp, Phoma exigua, Alternaria sp.

Les différents délais entre arrachage et mise en conservation n'ont manifesté que peu d'incidence sur la répartition de la mycoflore fongique. De même, on ne note pas d'influence sensible de la durée du stockage sur la part relative des principales espèces fongiques, si ce n'est une légère augmentation de la fréquence de $P$ megasperma et une mycoflore moins diversifiée après $100 \mathrm{j}$ de conservation.

\section{Mise en évidence de l'expression du pouvoir pathogène de $\mathrm{M}$ acerina et $\mathrm{B}$ cinerea dans les conditions optimales de conservation de la carotte}

L'homogénéité du comportement intraspécifique des champignons testés sur carotte permet de classer nos 20 isolats (tableau I) en 3 groupes en fonction de leur agressivité (fig 5) :

- le premier (nos 16-20 : Phoma exigua, Phomopsis sp, Alternaria sp et Cylindrocarpon sp) est composé d'isolats non pathogènes sur racines de carotte, quelle que soit la température étudiée;

- le second (nos 9-15: Sclerotinia sclerotiorum, Fusarium roseum, Stemphylium radicinum, Phytophthora megasperma) comprend des champignons agressifs sur carottes, mais seulement à partir de $5{ }^{\circ} \mathrm{C}$. À cette température, les niveaux d'attaque de $F$ roseum et de $S$ radicinum restent très faibles (10 et $30 \mathrm{~mm}^{2}$ de surface touchée), alors que $S$ sclerotiorum est le plus agressif de tous les champignons testés $\left(110 \mathrm{~mm}^{2}\right)$;

- le troisième (nos 1-8: $M$ acerina et $B$ cinerea) est le seul à provoquer des attaques dès $0,5^{\circ} \mathrm{C}$, avec des surfaces nécrosées pouvant atteindre $50 \mathrm{~mm}^{2}$ au bout de $6 \mathrm{j}$. La température optimale d'attaque de ces 2 espèces est au moins égale à $15^{\circ} \mathrm{C}$; à 10 comme à $15^{\circ} \mathrm{C}$, elles sont moins agressives que $S$ sclerotiorum. Enfin, il est intéressant de noter que les souches de $M$ acerina et $B$ cinerea $\left(n^{\circ} 1\right.$ et $n^{\circ} 5$ ) isolées au champ respectivement sur carotte et sur féverole, sont également capables de s'exprimer dès $0,5^{\circ} \mathrm{C}$.

\section{DISCUSSION ET CONCLUSION}

Les résultats présentés apportent des informations sur la pathologie de la carotte en cours de conservation et sur l'optimisation des techniques de stockage au froid.

Ainsi, ce travail met en évidence la relation existant entre les conditions de conservation et l'intensité des attaques parasitaires : une humidité relative proche de la saturation $(98 \% \mathrm{HR})$ associée à une température voisine de $1^{\circ} \mathrm{C}$ permet de limiter les problèmes parasitaires, ce qui est en accord avec les résultats obtenus au Canada et en Norvège dans des situations culturales tout à fait différentes (Van Den Berg et Lentz, 1973; Apeland, 1983).

Dans ces conditions de conservation considérées comme optimales (Villeneuve et al, 1992), l'aménagement d'un délai d'au moins $12 \mathrm{~h}$ entre la récolte et la mise en conservation permet de limiter significativement les pertes dues aux attaques parasitaires. Selon Lewis et al (1981), ce délai favorise la cicatrisation des blessures particulièrement favorables au développement de $M$ acerina (Davies et al, 1981). On peut penser qu'une température ambiante plus élevée lors 
A
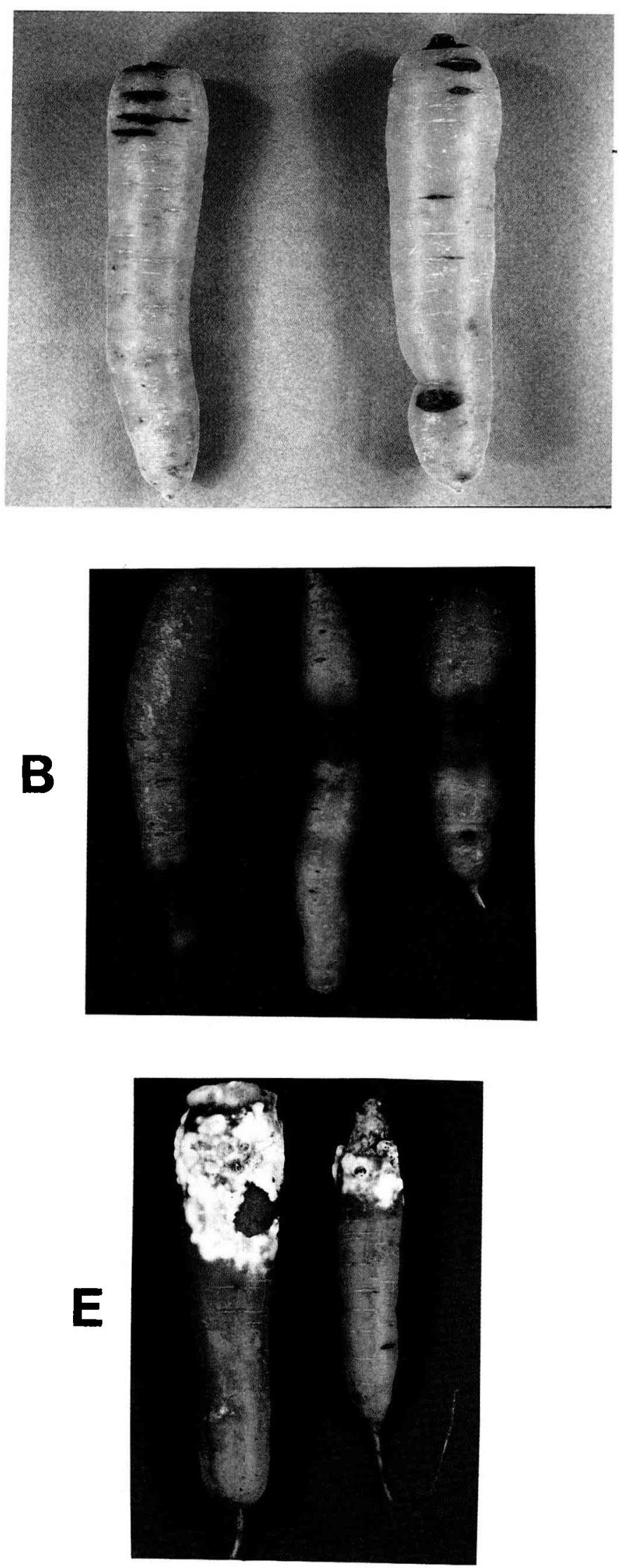

C
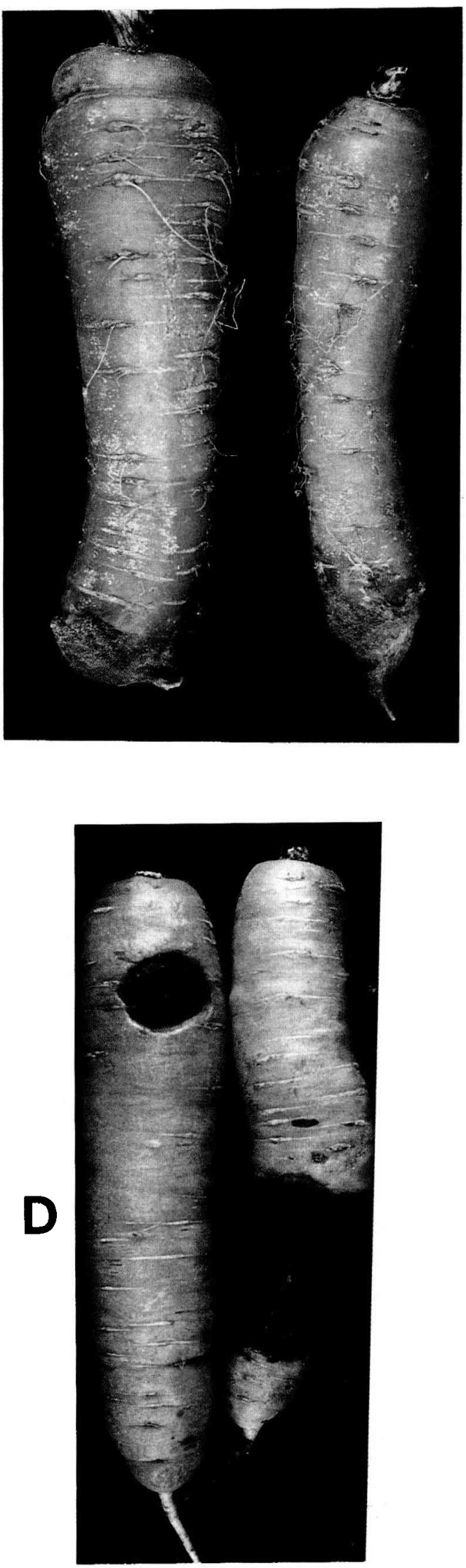

Fig 4. Symptômes des principales maladies rencontrées sur carottes conservées au froid; a : Mycocentrospora acerina; $b$ : Phytophthora megasperma; c : Botrytis cinerea; d: Stemphylium radicinum; e : Sclerotinia sclerotiorum. 


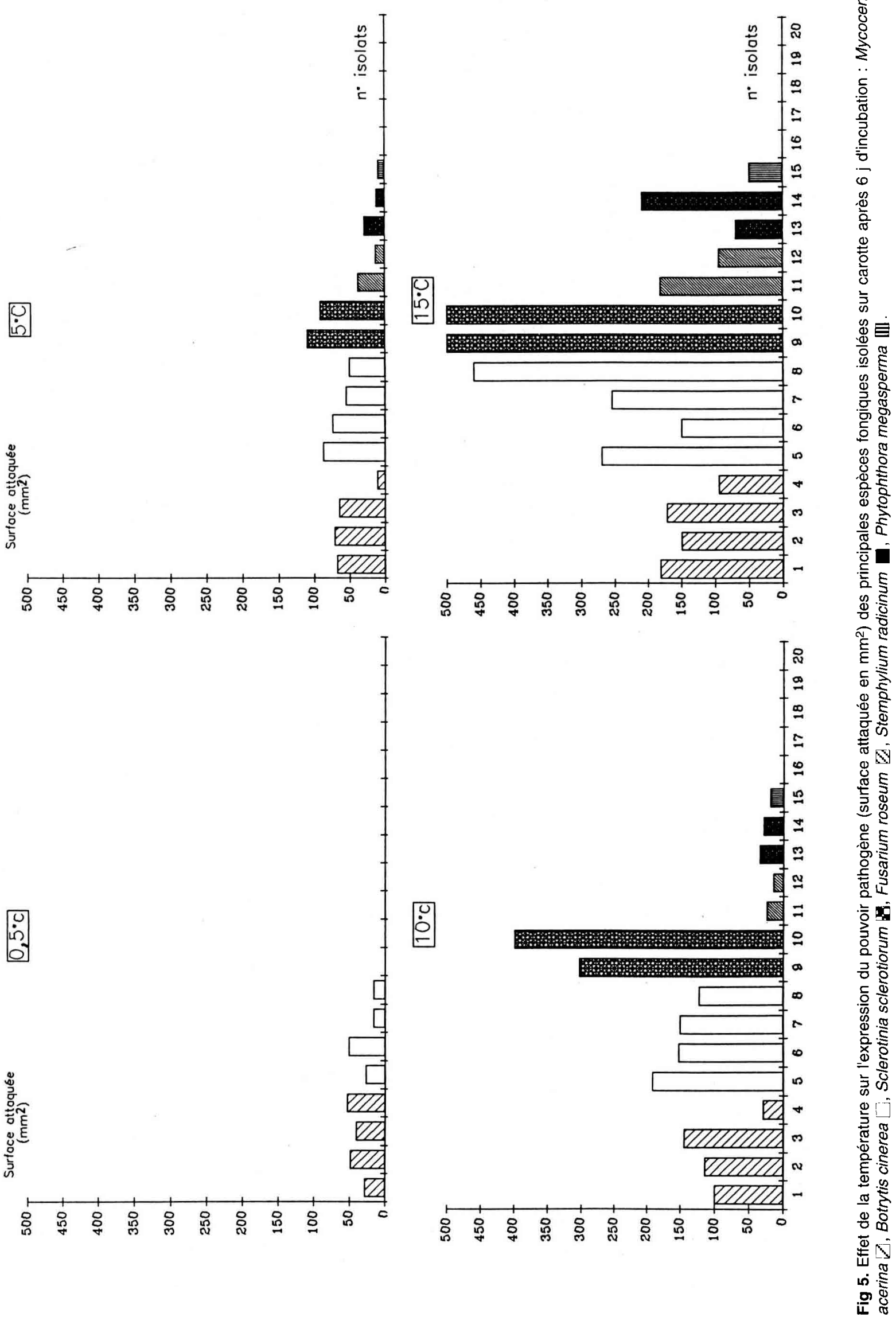


de la phase transitoire arrachage-mise en conservation aurait permis d'amplifier cet effet en activant à la surface des blessures la production de phytoalexines ( 6 Méthoxymelléine, Falcarindiol) intervenant dans les processus de cicatrisation (Lewis et al, 1981).

L'inventaire de la mycoflore, réalisé sur racines de carottes stockées au froid humide pendant 50 ou $100 \mathrm{j}$, révèle la présence très importante de $M$ acerina. Connu à l'étranger pour son incidence économique sur carottes conservées au froid (Lewis et Garrod, 1983), ce champignon n'avait jusqu'ici été signalé qu'occasionnellement en France en cours de culture (Breton et Rouxel, 1985a). Les inoculations réalisées au laboratoire ont confirmé l'agressivité de $M$ acerina dans les conditions optimales de conservation $\left(0,5^{\circ} \mathrm{C}\right)$. II est plus surprenant de constater de fortes attaques dues à $P$ megasperma, parasite plus redouté pendant la phase culturale (Breton et Rouxel, 1985b). L'absence de pathogénicité de $P$ megasperma à $0,5^{\circ} \mathrm{C}$ suggère d'ailleurs que les dégâts observés en conservation résultent vraisemblablement de l'évolution d'infections contractées avant la récolte mais ne présentant pas de symptômes à cette date. Bien que $S$ sclerotiorum et $S$ radicinum aient été décrits comme très préjudiciables sur carottes stockées au froid par de nombreux auteurs (Schwartz, 1977; Geary, 1978), ces 2 agents pathogènes souvent observés au champ ne se sont que très peu manifestés dans nos conditions d'étude; les inoculations réalisées au laboratoire ont mis en évidence leur perte d'agressivité à $0,5^{\circ} \mathrm{C}$. D'autre part, l'absence d'agressivité de Cylindrocarpon $\mathrm{sp}$, Phoma exigua, Phomopsis sp et Alternaria $\mathrm{sp}$ confirme l'existence sur carotte de tout un cortège de champignons déjà décrits au champ (Montfort et Rouxel, 1988) mais sans incidence notable sur la qualité de la conservation.

Parmi les autres constituants de la mycoflore associée aux symptômes, $F$ roseum apparaît également comme un parasite de conservation d'importance très secondaire, son agressivité ne s'exprimant qu'à partir de $5{ }^{\circ} \mathrm{C}$. Concernant $\mathrm{B}$ cinerea, les travaux de Goodliffe et Heale (1977) ont montré qu'une humidité relative proche de la saturation réduisait sensiblement ses attaques en limitant les pertes de poids, paramètre très favorable au développement de ce parasite. On peut donc admettre que nos conditions de conservation n'ont pas permis à ce parasite de se développer, bien que son agressivité à $0,5^{\circ} \mathrm{C}$ ait été mise en évidence. Enfin, nos résultats montrent que les souches pathogènes isolées au froid ne présentent pas d'adaptation à cet environnement particulier mais semblent n'être, au contraire, que le reflet de la microflore racinaire présente naturellement pendant la phase culture.

En conclusion, cette étude a permis de hiérarchiser l'importance des principaux agents pathogènes susceptibles de se manifester sur carottes stockées au froid; elle a, en particulier, montré le rôle prépondérant joué par un champignon jusqu'ici peu connu en France : Mycocentrospora acerina. Le rôle de plusieurs paramètres, liés à la phase pré- ou post-récolte, dans l'évolution de l'état sanitaire des racines en cours de conservation est maintenant bien établi : l'intérêt du stockage au froid humide et du respect d'un délai d'au moins $12 \mathrm{~h}$ entre arrachage et mise en conservation est clairement démontré; enfin, les produits issus de parcelles "à risques" se conservent mal, même en conditions de conservation optimisées.

II apparaît malgré tout, pour $M$ acerina, que même dans ces conditions optimales de conservation, les pertes demeurent élevées $(20 \%$ des racines atteintes), ce taux étant d'autant plus insupportable que les coûts de stockage au froid humide sont élevés. D'autres voies doivent donc être recherchées pour tenter de limiter ces pertes. Si une protection chimique peut être envisagée au champ avant la récolte, c'est sans doute à travers l'étude des relations hôteparasite et plus particulièrement la dynamique des réactions de défense de la carotte qu'une solution durable et raisonnée pourra être trouvée. Il est en effet établi que certains oligosaccharides d'origine pariétale, libérés lors d'infections parasitaires, sont capables d'induire l'accumulation de phytoalexines (Nothnagel et al, 1983) et d'autres réactions de défense chez la plante (Hahn et al, 1989). La mise en évidence de tels éliciteurs chez le couple Daucus carota- $M$ acerina devrait permettre à terme de déclencher ces mécanismes de défense et de définir des critères de sélection pour les généticiens.

\section{RÉFÉRENCES}

Apeland J (1983) Storage - Long and short terme. ADAS Carrot Conference. 18-20 April, session 3

Breton D, Rouxel F (1985a) Les pourritures hivernales de la carotte. Phytoma 373, 38-40

Breton D, Rouxel F (1985b) Étude des pourritures hivernales de la carotte: la maladie de la bague due au Phytophthora megasperma. CR $1^{\text {re }} \mathrm{J}$ études ma-ladies des plantes, 26 et 27 février. Ann Phytopathol 2, 165-173 
Davies WP, Lewis BG, Day JR (1981) Observations on infection of stored carrot roots by Mycocentrospora acerina. Trans Br Mycol Soc 77 (1), 139-151

Derbyshire DM, Crisp AF (1971) Vegetable storage diseases in East Anglia. Proc 6th Br Insect Fung Conf, Brighton, 167-172

Derbyshire DM, Crisp AF (1978) Studies on treatment to prolong the storage life of carrots. Exp Hortic 30 , 23-28

Geary J (1978) Host-parasite interactions between the cultivated carrot (Daucus carota L) and Sclerotinia sclerotiorum (Lib) de Bary. Ph D Thesis. Univ East Anglia, UK. $220 p$

Geeson JD, Browne KM, Everson Helen P (1988) Storage diseases of carrots in East Anglia 1978-1982, and the effects of some pre and post harvest factors. Ann Appl Biol 112, 503-514

Goodliffe JP, Heale JB (1977) Factors affecting the resistance of stored carrot roots to Botrytis cinerea. Ann Appl Biol 80, 243-246

Hahn MG, Bucheli P, Cervone F, Doares SH, O'Neil RA, Darvill A, Albersheim P (1989) The roles of cell wall constituents in plant-pathogen interactions. $I n$ : Plant-microbe interactions (Kosuge T, Nester EW, eds) Vol 3 McGraw hill, New York, 131-181

Hoftun $\mathrm{H}$ (1985) Testing of storage ability in carrots. XX Vortragstagung. 25-27 Mars 1985, FreisingWeihenstephan, 131-138

Jensen A (1969) Rhizoctonia carotae Rader, a new and important pathogen to carrots in Denmark. Friesia $9,84-92$

Lewis BG, Garrod B (1983) Carrots. In: Post-harvest pathology of fruits and vegetables (Collins, Dennis, eds). Acad Press, London 218-257

Lewis BG, Davies WP, Garrod B (1981) Wound healing in carrot crops in relation to infection by $\mathrm{Myco}$ centrospora acerina. Ann App/ Bio/ 99, 35-42
Montfort F, Rouxel F (1986) La maladie de la tache de la carotte. Phytoma 376, 43-45

Montfort F, Rouxel F (1988) La maladie de la "tache» de la carotte due à Pythium violae Chesters et Hickman : données symptomatologiques et étiologiques. Agronomie 8 (8), 701-706

Nothnagel EA, McNeil M, Albersheim P, Dell A (1983) Host-pathogen interactions. XXII. A galacturonic acid oligosaccharide from plant cell walls elicits phytoalexins. Plant Physiol 71, 916-926

Punja ZK (1987) Mycelia growth and pathogenesis by Rhizoctonia carotae on carrot. Can J Plant Pathol 9, 24-31

Rader WE (1948) Rhizoctonia carotae $\mathrm{n} \mathrm{sp}$ and Gliocladium aureum $n \mathrm{sp}$, two news pathogens of carrots in cold storage. Phytopathology 38, 400452

Ramert B (1984) New postharvest diseases of carrots. Rhizoctonia carotae and Mycocentrospora acerina. Vaxtskyddsnotiser 48, 110-112

Schwartz A (1977) Conservation de la carotte. Relation possible entre la conservabilité et les conditions au champ. Acta Hortic 62, 345-350

Tahvonen R (1985) The prevention of Botrytis cinerea and Sclerotinia sclerotiorum on carrots during storage by spraying the tops with fongicides before harvesting. Ann Agric Fenn 24 (2), 8995

Tronsmo A (1989) Trichoderma harzianum used for biological control of storage rot on carrots. Norw $J$ Agric Sci 3, 157-161

Villeneuve F, Luneau C, Bosc JP (1992) Conservation de la carotte en chambre froide. Fruits Légumes $93,69-71$

Van Den Berg L, Lentz CP (1973) High humidity storage of carrots, parsnips, rutabagas and cabbage. J Am Soc Hortic Sci 98, 129-132 